\title{
PROPOSITION DE PRÉCONDITIONNEURS PSEUDO-DIFFÉRENTIELS POUR L'ÉQUATION CFIE DE L'ÉLECTROMAGNÉTISME
}

\author{
DAVID P. LEVADOUX ${ }^{1}$
}

\begin{abstract}
Résumé. On exhibe dans cette note une paramétrix (au sens faible) de l'opérateur sous-jacent à l'équation CFIE de l'électromagnétisme. L'intérêt de cette paramétrix est de se prêter à différentes stratégies de discrétisation et ainsi de pouvoir être utilisée comme préconditionneur de la CFIE. On montre aussi que l'opérateur sous-jacent à la CFIE satisfait une condition Inf-Sup discrète uniforme, applicable aux espaces de discrétisation usuellement rencontrés en électromagnétisme, et qui permet d'établir un résultat inédit de convergence numérique de la CFIE.
\end{abstract}

\begin{abstract}
We present a weak parametrix of the operator of the CFIE equation. An interesting feature of this parametrix is that it is compatible with different discretization strategies and hence allows for the construction of efficient preconditioners dedicated to the CFIE. Furthermore, one shows that the underlying operator of the CFIE verifies an uniform discrete Inf-Sup condition which allows to predict an original convergence result of the numerical solution of the CFIE to the exact one.
\end{abstract}

Classification Mathématique. 15A12, 65F10, 65N12, 65R20, 65Z05.

Reçu le 7 juin 2004. Revisé le 6 décembre 2004.

\section{INTRODUCTION}

L'équation CFIE (Combined Field Integral Equation) est une équation très populaire, que résolvent la plupart des codes industriels dédiés à la modélisation électromagnétique. Une raison essentielle de ce succès est que la CFIE est une équation sans résonnance, c'est-à-dire admet une et une seule solution, et ce quelle que soit la fréquence. Autrement dit, il n'existe pas de fréquence irrégulière comme pour l'équation EFIE (Electric Field Integral Equation), autre équation fameuse mais qui, appliquée à des surfaces fermées, dégénère en des points de fréquences de plus en plus rapprochés lorsque la fréquence augmente. Pour cette raison, c'est souvent la CFIE que l'on privilégie lors d'un calcul à haute fréquence ( $c f$. les thèses sur les méthodes multipôles rapides $[5,16$, 20]). Cependant, l'expérience montre aussi que certains calculs de très grandes tailles dépassant le million de degrés de liberté (la section efficace radar d'un avion à plusieurs $\mathrm{GHz}$ par exemple), ne sont que très difficilement résolus (voire pas du tout...) par la CFIE, en raison d'une convergence beaucoup trop lente, et ce malgré l'utilisation de préconditionneurs. Ainsi, la recherche de préconditionneurs efficaces revêt une importance stratégique, et la littérature dans ce domaine est riche en propositions. Cependant, les préconditionneurs utilisés en milieu industriel sont souvent de nature algébrique, c'est-à-dire construits à partir de la matrice de Galerkin de l'équation discrétisée, sans souci des propriétés mathématiques de l'équation continue. Or, des préconditionneurs

Mots Clés. Preconditioner, integral equation, electromagnetism, pseudodifferential operator, numerical analysis.

1 ONERA, centre de Palaiseau, Chemin de la Hunière, 91761 Palaiseau, France. david.levadoux@onera.fr 
«plus intelligents » existent pourtant. On peut citer par exemple la technique présentée dans [9] ou [11] qui, à partir de l'analyse symbolique des projecteurs de Calderón, propose une famille d'équations régularisant les équations de type CFIE rencontrées en acoustique, et dont la généralisation aux équations de Maxwell, produit des systèmes linéaires très bien conditionnés [10,13]. Toujours grâce aux propriétés des projecteurs de Calderón, on trouve aussi dans [6] et [1] des méthodes pour construire des préconditionneurs de l'EFIE qui semblent très performants.

Cependant, dans le cas de la CFIE, la recherche d'un préconditionneur se heurte à la difficulté que les formules de Calderón ne permettent pas de trouver une paramétrix de l'opérateur sous-jacent à cette équation, contrairement à ce qui survient pour l'EFIE. Le travail que l'on présente dans cette note se propose donc d'exhiber une telle paramétrix, à partir de laquelle peuvent être imaginés divers préconditionneurs.

Après un bref rappel de la structure de l'équation CFIE dans la partie 2, on exhibe dans la partie 3 une paramétrix $B$ (au sens faible) de l'opérateur $A$ sous-jacent à la CFIE. Les outils principaux pour établir ce résultat sont conjointement, la décomposition de Helmholtz et le calcul pseudo-différentiel. Ce résultat exprime notamment que le produit $B A$ est égal à l'identité, modulo une perturbation compacte $C$. Or, il est bien connu que la discrétisation d'un opérateur inversible de la forme $1+C$ conduit à un système linéaire $[1+C]$ bien conditionné. Il est donc naturel de penser pouvoir utiliser la discrétisation $[B]$ de $B$ comme préconditionneur de $[A]$. On étudie donc dans la partie 4 deux façons de discrétiser $B$ de telle sorte que le produit $[B][A]$ soit, en un certain sens et asymptotiquement en $h$ (finesse du maillage), une approximation convergente de $[B A]=[1+C]$. On espère ainsi faire hériter $[B][A]$ du conditionnement favorable propre à $[1+C]$.

Signalons enfin que ce travail a permis d'établir au passage un résultat inédit. L'inégalité Inf-Sup discrète uniforme en $h$ pour $A$, contenue dans la proposition 4.2, prouve en effet que la solution numérique de la CFIE converge vers la solution exacte, en énergie pour les courants et en norme $H^{-1 / 2}$ pour les charges. C'est à la connaissance de l'auteur, la seule analyse numérique de la convergence qui existe à ce jour pour la CFIE.

\section{L'ÉqUATION CFIE DE L'ÉLECTROMAGnÉTisme}

On se donne un compact $D$ de $\mathbb{R}^{3}$ dont le complémentaire $\Omega$ est connexe et dont la frontière $\Gamma$ est une variété de régularité $\mathcal{C}^{\infty}$ difféomorphe à une sphère. La normale unitaire sortante sur $\Gamma$ est notée $\nu$, et l'opérateur trace tangentielle est noté $\gamma_{\mathrm{T}}$. On rappelle que le potentiel vecteur $G$ (dépendant d'un nombre réel $k$ ) associe à tout champ de vecteurs $u$ tangents sur $\Gamma$, le champ vectoriel défini pour tout $x \in \mathbb{R}^{3} \backslash \Gamma$ par :

$$
G u(x)=\frac{-1}{4 \pi} \int_{\Gamma} \frac{\mathrm{e}^{i k\|x-y\|}}{\|x-y\|} u(y) \mathrm{d} y,
$$

où \|\| désigne la norme euclienne sur $\mathbb{R}^{3}$. Les traces de $G$ définissent alors deux opérateurs de frontière :

$$
J_{1}=-i \nu \times\left(k G+\frac{1}{k} \nabla G \nabla \cdot\right) \quad J_{2}=\nu \times \nabla \times G+\frac{1}{2}
$$

qui sont pseudo-différentiels, d'ordre respectivement 1 et $-1(\nu \times, \nabla \cdot, \nabla, \nabla \times$ désignant respectivement l'opérateur trace tangentielle sur $\Gamma$ suivi du produit vectoriel avec $\nu$, le divergent surfacique, le gradient et le rotationel volumiques).

L'équation intégrale que l'on cherche à préconditionner résout un problème aux limites classique en électromagnétisme. Il s'agit de trouver l'unique champ électrique $E$ solution de $\nabla \times \nabla \times E-k^{2} E=0$ sur $\Omega$, et vérifiant à la fois une condition de rayonnement à l'infini de type Silver-Müller, et la condition au bord $\gamma_{\mathrm{T}} E=-E^{i}$, où $E^{i}$ est un champ électrique que l'on se donne sur un voisinage ouvert de $D$. Ce problème modélise par exemple la diffraction d'un champ électrique par un corps parfaitement conducteur. 
L'équation CFIE, d'inconnue une distribution $j$ sur $\Gamma$, s'écrit $A j=\alpha \gamma_{\mathrm{T}} E^{i}+\beta \frac{1}{i k} \nu \times \nabla \times E^{i}$, où $\alpha$ et $\beta$ sont des réels arbitraires positifs non nuls, et

$$
A=\alpha \nu \times J_{1}+\beta\left(\frac{1}{2}+J_{2}\right) .
$$

Des résultats classiques établissent que le noyau de $A$, cherché dans des espaces de Sobolev ou de Hölder suffisamment réguliers, est trivial [7].

Pour toute la suite, on adopte les notations suivantes. L'espace des fonctions numériques définies sur $\Gamma$ (resp. des champs de vecteurs tangents à $\Gamma$ ) de régularité $\mathcal{C}^{\infty}$ est noté $\mathcal{D}(\Gamma)\left(\right.$ resp. $\mathcal{D}_{\mathrm{T}}(\Gamma)$ ). La norme d'un espace de Sobolev $H^{s}$ (scalaire ou vectoriel) est écrite ||$_{s}$ ou simplement || si $s=0$. Le produit hermitien de $H^{0}$ est noté ( , ). On note $H_{\mathrm{T}}^{s}$ l'espace des champs vectoriels tangents à $\Gamma$ et de régularité $H^{s}$, et $H_{\mathrm{div}}^{s}$ les champs de $H_{\mathrm{T}}^{s}$ dont les divergents surfaciques sont de régularité $H^{s}$. La norme d'un élément $u$ de $H_{\text {div }}^{s}$ est $\|u\|_{s}=|u|_{s}+|\nabla \cdot u|_{s}$, et si $s=0$ on écrit encore simplement $\|u\|$. Le gradient et le divergent surfacique sont respectivement notés $\nabla$ et $\nabla \cdot$.

\section{UNE PARAMÉTRIX POUR LA CFIE}

Un résultat important (démontré par exemple dans [4]), énonce que $H_{\mathrm{T}}^{0}(\Gamma)$ est somme directe de deux sousespaces fermés et orthogonaux entre eux :

$$
H_{\mathrm{T}}^{0}(\Gamma)=\nu \times \nabla\left(H^{1}(\Gamma)\right) \oplus^{\perp} \nabla\left(H^{1}(\Gamma)\right) .
$$

Cette décomposition est connue sous le nom de décomposition de Helmholtz. Il est possible d'expliciter les projecteurs qui lui sont associés à l'aide du pseudo-inverse (au sens de Moore-Penrose) de l'opérateur de LaplaceBeltrami. Pour ce faire, on décompose l'espace $\mathcal{D}(\Gamma)$ en la somme directe $\mathcal{D}(\Gamma)^{\bullet} \oplus C$, où $\mathcal{D}(\Gamma)^{\bullet}=\{f \in$ $\left.\mathcal{D}(\Gamma) ; \int_{\Gamma} f(x) \mathrm{d} x=0\right\}$ et $C$ est l'espace des fonctions constantes. On note $\Pi: \mathcal{D}(\Gamma) \rightarrow \mathcal{D}(\Gamma)^{\bullet}$ le premier projecteur relatif à cette décomposition qui s'écrit $\Pi f=f-\int_{\Gamma} f(x) \mathrm{d} x /|\Gamma|$, où $|\Gamma|$ est la mesure de $\Gamma$. Puisque le laplacien surfacique $\Delta$ induit un difféomorphisme $\widetilde{\Delta}$ de $\mathcal{D}(\Gamma)^{\bullet}$ sur lui-même, le pseudo-inverse $\Delta^{-1}$ de $\Delta$ est défini par $\Delta^{-1}=\widetilde{\Delta}^{-1} \Pi$. Cet opérateur est pseudo-différentiel, de symbole principal $-\|\xi\|^{-2}\left(\xi \in T^{\star} \Gamma\right.$, fibré vectoriel cotangent de $\Gamma$ ), et permet de clarifier la nature des projecteurs sous-jacents à la décomposition de Helmholtz :

Proposition 3.1. Les projecteurs $\left(\Pi_{1}, \Pi_{2}\right)$ de la décomposition de Helmholtz (3) s'écrivent :

$$
\Pi_{1}=-\nu \times \nabla \Delta^{-1} \nabla \cdot \nu \times \quad \Pi_{2}=\nabla \Delta^{-1} \nabla \cdot
$$

Preuve. Notons $\left(\pi_{1}, \pi_{2}\right)$ les projecteurs sous-jacents à la décomposition (3). Pour tout $u=\nu \times \nabla f$ avec $f \in H^{1}(\Gamma)$ on a $\Pi_{1} u=\nu \times \nabla \Delta^{-1} \nabla \cdot \nabla f=\nu \times \nabla\left(f-\int_{\Gamma} f(x) \mathrm{d} x /|\Gamma|\right)=\nu \times \nabla f=u$. Donc $\Pi_{1} \pi_{1}=\pi_{1}$, et puisque $\Pi_{1} \pi_{2}=0$ il vient que $\Pi_{1}=\Pi_{1}\left(\pi_{1}+\pi_{2}\right)=\pi_{1}$. On démontre de façon analogue que $\Pi_{2}=\pi_{2}$.

À partir de maintenant et pour toute la suite, la notation $G$ (resp. $G_{0}$ ) désigne la trace tangentielle du potentiel vecteur (1) pour $k \neq 0$ (resp. $k=0$ ). Les opérateurs de frontière $G$ ou $G_{0}$ ont alors pour symbole principal[9] :

$$
\sigma_{G}(\xi)=\sigma_{G_{0}}(\xi)=-\|\xi\|^{-1} / 2 \quad\left(\xi \in T^{\star} \Gamma\right)
$$

Proposition 3.2. L'opérateur A défini en (2) et l'opérateur B défini par:

$$
B=\frac{i 4 k}{\alpha} G_{0}+\frac{2}{\beta} \Pi_{1} .
$$


sont tels que les symboles principaux des produits $A B$ et $B A$ valent 1 :

$$
\sigma(A B)=\sigma(B A)=1
$$

Preuve. On remarque que modulo $\Psi^{-1}(\Gamma)$ (classe des opérateurs pseudo-différentiels d'ordre -1 ), on a les équivalences :

$$
\nu \times J_{1} \Pi_{1} \sim 0 \quad \nabla G \nabla \cdot G_{0} \sim-\frac{\Pi_{2}}{4} .
$$

L'équivalence de gauche est conséquence du fait que $\nabla \cdot \Pi_{1}=0$ et que $G$ est d'ordre -1 , celle de droite, que les opérateurs sont d'ordre 0 et ont même symbole principal (sachant (5), le calcul est trivial en revenant à la formule explicite (4) décrivant $\Pi_{2}$ ). Grâce aux équivalences (8) on peut alors mener le calcul suivant, modulo $\Psi^{-1}(\Gamma)$ :

$$
\begin{aligned}
A B & \sim\left[\alpha \nu \times J_{1}+\beta\left(\frac{1}{2}+J_{2}\right)\right]\left[\frac{i 4 k}{\alpha} G_{0}+\frac{2}{\beta} \Pi_{1}\right] \\
& \sim i 4 k \nu \times J_{1} G_{0}+2\left(\frac{1}{2}+J_{2}\right) \Pi_{1} \sim-4 \nabla G \nabla \cdot G_{0}+\Pi_{1} \sim \mathrm{Id}
\end{aligned}
$$

ce qui prouve que $\sigma(A B)=1$. On démontre de la même façon que $\sigma(B A)=1$.

La relation (7) prouve que $A$ et $B$ sont hypoelliptiques. Par exemple, si $A u=v$ avec $v \in \mathcal{D}_{\mathrm{T}}(\operatorname{champs}$ de vecteurs $\mathcal{C}^{\infty}$ tangents à $\Gamma$ ) alors il existe un opérateur $C$ d'ordre $\leq-1$ tel que $u=C u+B v$. Puisque $C u \in H_{\mathrm{T}}^{s+1}$ si $u \in H_{\mathrm{T}}^{s}$, et que $B v \in \mathcal{D}_{\mathrm{T}}$, on en déduit que $u \in \mathcal{D}_{\mathrm{T}}$.

D'autre part, comme le noyau de $A$ est trivial dans $\mathcal{D}_{\mathrm{T}}$, l'hypoellipticité de $A$ permet de conclure que son noyau est encore trivial dans $\mathcal{D}_{\mathrm{T}}^{\prime}$. Vérifions que c'est aussi le cas pour $B$.

Si l'on se donne $u \in \mathcal{D}_{\mathrm{T}}$, alors $\left(\Pi_{1} u, u\right)=\left|\Pi_{1} u\right|^{2}$ et $\Im \mathrm{m}(B u, u)=\frac{4 k}{\alpha}\left(G_{0} u, u\right)$. Or d'après [14], il existe une constante $C>0$ indépendante de $u$ telle que :

$$
\left(G_{0} u, u\right) \geq C|u|_{-1 / 2}^{2}
$$

Par conséquent, le noyau de $B$ est trivial sur $\mathcal{D}_{\mathrm{T}}$ et donc aussi sur $\mathcal{D}_{\mathrm{T}}^{\prime}$ par hypoellipticité.

On introduit maintenant les espaces fonctionnels adaptés à notre problème. Pour tout $s \in \mathbb{R}$ on pose :

$$
\mathcal{L}^{s}=\left\{u \in H_{\mathrm{T}}^{s} ; \nabla \cdot u=0\right\} \quad \mathcal{S}^{s}=\left\{u \in H_{\mathrm{T}}^{s} ; \nabla \cdot \nu \times u=0\right\} \quad \mathcal{H}^{s, s^{\prime}}=\mathcal{L}^{s}+\mathcal{S}^{s^{\prime}}
$$

Il va de soi que $\mathcal{L}^{s}$ et $\mathcal{S}^{s}$ munis de la norme $H_{\mathrm{T}}^{s}$ sont des espaces de Hilbert, de même que $\mathcal{H}^{s, s^{\prime}}$ si on le munit de la norme $\left(\left|\Pi_{1} u\right|_{s}^{2}+\left|\Pi_{2} u\right|_{s^{\prime}}^{2}\right)^{1 / 2}$. On est à présent en mesure de démontrer le résultat central suivant :

Théorème 3.3. Si $s, s^{\prime}$ sont des réels tels que $s^{\prime}<s$ et $\left|s^{\prime}-s\right|<1$, alors les opérateurs $A$ et $B$ réalisent les continuités suivantes de façon bijective :

$$
A: \mathcal{H}^{s, s^{\prime}+1}(\Gamma) \rightarrow \mathcal{H}^{s, s^{\prime}}(\Gamma) \quad B: \mathcal{H}^{s, s^{\prime}}(\Gamma) \rightarrow \mathcal{H}^{s, s^{\prime}+1}(\Gamma) .
$$

Preuve. On remarque que tout opérateur d'ordre 0 est continu de $\mathcal{H}^{s, s^{\prime}+1}$ dans $H_{\mathrm{T}}^{s}$ qui est inclus continûment dans $\mathcal{H}^{s, s^{\prime}}$. De même, tout opérateur d'ordre -1 est continu de $\mathcal{H}^{s, s^{\prime}}$ dans $H_{\mathrm{T}}^{s^{\prime}+1}$ qui est inclus continûment dans $\mathcal{H}^{s, s^{\prime}+1}$. Il suffit donc d'établir les continuités pour $\nabla G \nabla \cdot$ et $\Pi_{1}$. La relation $\nabla G \nabla \cdot\left(\Pi_{1}+\Pi_{2}\right)=\nabla G \nabla \cdot \Pi_{2}$ montre que $\nabla G \nabla$. est continu de $\mathcal{H}^{s, s^{\prime}+1}$ dans $\mathcal{S}^{s^{\prime}}$, et donc $A$ est continu de $\mathcal{H}^{s, s^{\prime}+1}$ dans $\mathcal{H}^{s, s^{\prime}}$. Quant à $\Pi_{1}$, il est continu de $\mathcal{H}^{s, s^{\prime}}$ dans $\mathcal{L}^{s}$ et donc $B$ est continu de $\mathcal{H}^{s, s^{\prime}}$ dans $\mathcal{H}^{s, s^{\prime}+1}$.

Démontrons maintenant les bijectivités grâce à la théorie de Fredholm. Puisque tout opérateur d'ordre -1 est compact indifféremment dans $\mathcal{H}^{s, s^{\prime}}$ ou $\mathcal{H}^{s, s^{\prime}+1}$, la relation $(7)$ implique que $A B$ et $B A$ sont des perturbations compactes de l'identité, et donc des opérateurs de Fredholm d'index 0. Or, les noyaux de $A$ et $B$ sont triviaux, donc ceux de $A B$ et $B A$ aussi. Donc $A B$ et $B A$ sont bijectifs, ainsi que $A$ et $B$. 


\section{Des préconditionneurs pour la CFIE}

\subsection{Notations et cadre fonctionnel}

On pose $X=\mathcal{H}^{0,1 / 2}$ et $Y=\mathcal{H}^{0,-1 / 2}$. Chacun de ces espaces étant dual de l'autre autour de l'espace pivot $H_{\mathrm{T}}^{0}$ muni du produit scalaire $\langle u, v\rangle=(u, \bar{v})$, les opérateurs $A$ et $B$ réalisent les continuités $A: X \rightarrow X^{\star}$ et $B: Y \rightarrow Y^{\star}$. On note $M: X \rightarrow Y^{\star}$ l'isomorphisme qui à tout $u \in X$ associe la forme $v \mapsto\langle u, v\rangle$. L'opérateur transposé de $M$ qui est égal à $M$ dans la théorie des distributions (i.e. $M$ et ${ }^{t} M$ ont même noyau de Schwartz) est encore noté $M$.

Pour toute la suite, $\left(X_{h}\right)_{h>0}$ désigne une famille de sous-espaces de $X$, de dimensions finis, dont la vocation est d'approcher $X$ lorsque $h$ tend vers 0. D'une façon générale, lorsque $E$ est un espace de Banach contenant tous les $X_{h}$, on dira que $X_{h}$ approche cet espace fonctionnel si $\forall u_{0} \in X \cap E, \lim _{h \mapsto 0}\left(\inf _{u \in X_{h} \cap E}\left|u_{0}-u\right|_{X}\right)=0$.

Si $F$ est un autre espace de Banach contenant $X_{h}$, et $L: E \rightarrow F^{\star}$ un morphisme continu, alors on note $\mathcal{L}_{h}: X_{h} \rightarrow X_{h}^{\star}$ le morphisme canonique induit par $L$. Plus précisément, si $i_{h}$ désigne indifféremment l'injection canonique de $X_{h}$ dans $E$ ou celle de $X_{h}$ dans $F$, on obtient :

$$
\mathcal{L}_{h}=i_{h}^{\star} L i_{h}
$$

Lorsque $X_{h}$ hérite de la norme de $E$ et $X_{h}^{\star}$ de celle de $F^{\star}$, la norme d'opérateur de $\mathcal{L}_{h}$ et sa constante LBB sont notées respectivement $\left|\mathcal{L}_{h}\right|_{E F^{*}}$ et $\left[\mathcal{L}_{h}\right]_{E F^{\star}}\left(\right.$ i.e. si $\left.|u|_{E}=1,\left[\mathcal{L}_{h}\right]_{E F^{\star}} \leq\left|\mathcal{L}_{h} u\right|_{F^{\star}} \leq\left|\mathcal{L}_{h}\right|_{E F^{\star}}\right)$. Enfin, la matrice de Galerkin de $L$ associée à une base $\left(e_{i}^{h}\right)_{i}$ de $X_{h}$ est notée $L_{h}$. On peut définir $L_{h}$ par ses coefficients $\left(L_{h}\right)_{i j}=\left\langle L_{h} e_{j}^{h}, e_{i}^{h}\right\rangle_{F^{\star} \times F}$, ou plus abstraitement via le morphisme bijectif d'espace vectoriel $j_{h}: \mathbb{C}^{N(h)} \rightarrow X_{h}$ défini par $\lambda \mapsto \sum_{i} \lambda_{i} e_{i}^{h}\left(N(h)\right.$ étant la dimension de $\left.X_{h}\right)$. En identifiant canoniquement $\left(\mathbb{C}^{N(h)}\right)^{\star}$ à $\mathbb{C}^{N(h)}$, la matrice de Galerkin, vue comme endomorphisme de $\mathbb{C}^{N(h)}$, s'écrit alors :

$$
L_{h}=j_{h}^{\star} \mathcal{L}_{h} j_{h}
$$

La projection au sens $L^{2}$ de $X$ sur $X_{h}$ est notée $Q^{h}$ (i.e. $\left\langle u, u_{h}\right\rangle=\left\langle Q^{h} u, u_{h}\right\rangle, \forall u \in X, \forall u_{h} \in X_{h}$ ). Munissant $X_{h}$ de la norme de $X$, on démontre sans difficulté que $Q^{h}: X \rightarrow X_{h} \subset X$ reste continue sur $X$. De même, munissant $X_{h}$ de la norme de $Y, Q^{h}$ est prolongeable en une application continue sur $Y$ à valeurs dans $X_{h} \subset Y$. Lorsque les normes d'opérateurs $\left(\left|Q^{h}\right|_{X}\right)_{h>0}\left(\right.$ resp. $\left.\left(\left|Q^{h}\right|_{Y}\right)_{h>0}\right)$ sont bornées, on dit que $Q^{h}$ est stable sur $X$ (resp. $Y$ ). Cette propriété de stabilité est cruciale pour l'analyse numérique des préconditionneurs qui nous intéressent, mais on la retrouve aussi de façon prégnante dans la littérature ou elle est perçue comme reflet de la régularité du maillage sous-jacent à $X_{h}$ ( $c f$. par exemple [17,19] ou [18]). La proposition qui suit met donc en lumière le polymorphisme de cette propriété qui peut s'exprimer sous différentes formes.

Proposition 4.1. Les assertions suivantes sont toutes équivalentes :
i) $Q^{h}$ est stable sur $X$
ii) $Q^{h}$ est stable sur $Y$
iii) $\exists C>0, \forall h>0,\left[\mathcal{M}_{h}\right]_{X Y^{\star}}>C$
iv) $\exists C>0, \forall h>0,\left[\mathcal{M}_{h}\right]_{Y X^{\star}}>C$.

Preuve. La preuve résulte de la décomposition de $Q^{h}$ en le produit d'opérateurs suivant :

$$
Q^{h}=\mathcal{M}_{h}^{-1} i_{h}^{\star} \mathcal{M}
$$

où l'on rappelle que $i_{h}$ désigne l'injection canonique de $X_{h}$ dans $X$ ou $Y$. En effet, si par exemple $u \in Y$ alors pour tout $u_{h} \in X_{h}\left\langle\mathcal{M}_{h}^{-1} i_{h}^{\star} \mathcal{M} u, u_{h}\right\rangle=\left\langle\mathcal{M}_{h}\left(\mathcal{M}_{h}^{-1} i_{h}^{\star} \mathcal{M} u\right), u_{h}\right\rangle_{X_{h}^{\star} \times X_{h}}=\left\langle i_{h}^{\star} \mathcal{M} u, u_{h}\right\rangle_{X_{h}^{\star} \times X_{h}}=\left\langle\mathcal{M} u, i_{h} u_{h}\right\rangle_{X^{\star} \times X}=$ $\left\langle u, i_{h} u_{h}\right\rangle=\left\langle u, u_{h}\right\rangle$ et donc (13) est vraie. Par conséquent, puisque $\left|i_{h}^{\star}\right|_{X^{\star}}=1$ et $\left|\mathcal{M}_{h}^{-1}\right|_{X^{\star} Y}=\left[\mathcal{M}_{h}\right]_{Y X^{\star}}^{-1}$ on a $\left|Q^{h}\right|_{Y} \leq\left[\mathcal{M}_{h}\right]_{Y X^{\star}}^{-1}|\mathcal{M}|_{Y X^{\star}}$. D'autre part, $\left|Q^{h}\right|_{Y} \geq\left|\mathcal{M}_{h}^{-1} i_{h}^{\star}\right|_{X^{\star} Y}[\mathcal{M}]_{Y X^{\star}}$. Or $\left|\mathcal{M}_{h}^{-1} i_{h}^{\star}\right|_{X^{\star} Y}=\left|\mathcal{M}_{h}^{-1}\right|_{X^{\star} Y}$, car l'image par $i_{h}^{\star}$ de la boule unité fermée de $X^{\star}$ est la boule unité fermée de $X_{h}^{\star}$ (muni de la norme induite par la surjection $i_{h}^{\star}$ ). On a donc $\left|Q^{h}\right|_{Y} \geq\left[\mathcal{M}_{h}\right]_{Y X^{\star}}^{-1}[\mathcal{M}]_{Y X^{\star}}$. Ainsi ii) est équivalent à iv). On montrerait de la même 
manière que i) est équivalent à iii). Enfin, l'équivalence entre iii) et iv) est triviale car $\left[\mathcal{M}_{h}\right]_{X Y^{\star}}=\left[\mathcal{M}_{h}\right]_{Y X^{\star}}$, ce qui achève la preuve.

On suppose désormais que $X_{h}$ vérifie les hypothèses suivantes :

(H0) $X_{h}$ est stable par conjugaison : $u \in X_{h} \Rightarrow \bar{u} \in X_{h}$.

(H1) $X_{h}$ approche $X$ et $\mathcal{L}^{0}$ (courants d'énergies finies à divergence nulle).

(H2) La projection $Q^{h}$ au sens $L^{2}$ de $X$ sur $X_{h}$ est stable.

Si $X_{h}$ réalise la décomposition de Helmholtz (i.e. $\Pi_{1}$ et $\Pi_{2}$ restent des projecteurs de $X_{h}$ ) et si $X_{h}$ approche $X$, alors $X_{h}$ vérifie (H1) et (H2). C'est le cas par exemple des éléments proposés par Ivakhnenko et al.[8] qui satisfont en outre l'hypothèse (H0), de même que les éléments en cours d'implémentation dans un code de l'ONERA [21] dédié aux géométries axisymétriques. Christiansen a quant à lui démontré dans [6] que les éléments finis de Raviart-Thomas [15], fréquemment rencontrés dans les codes industriels, satisfont bien les hypothèses (H0) et (H1). Cependant, la question de savoir si de tels éléments satisfont (H2) est encore ouverte et semble délicate à trancher.

\subsection{Construction des préconditionneurs}

Adoptant la stratégie exposée par McLean et Tran [12] on cherche à construire des systèmes linéaires préconditionnés proches de $M_{h}^{-1}(B A)_{h}$ dont le conditionnement spectral est réputé bon puisqu'il converge vers celui de $B A$ qui est une perturbation compacte de l'identité inversible. Cependant, le calcul numérique de la matrice de Galerkin $(B A)_{h}$ ne peut se faire de façon classique, en intégrant le noyau de l'opérateur $B A$, puisque ce noyau n'est pas explicite. Mais si $B_{h}$ est calculable numériquement (par exemple si $X_{h}$ admet une décomposition de Helmholtz exacte), alors on suggère d'approcher $(B A)_{h}$ par $\left(B Q^{h} A\right)_{h}=B_{h} M_{h}^{-1} A_{h}$. On démontre cette égalité en remarquant que $B Q^{h} A=B i_{h} Q^{h} \mathcal{M}^{-1} A$ qui est égal à $B i_{h} \mathcal{M}_{h}^{-1} i_{h}^{\star} A$ d'après $(13)$, et en poursuivant le calcul en utilisant (11) et (12).

En revanche, si $B_{h}$ n'est pas directement calculable, on préférera approcher $(B A)_{h}$ plutôt par $\tilde{B}_{h} M_{h}^{-1} A_{h}$, où $\tilde{B}=i 4 k G_{0} / \alpha+2 \Pi_{1}^{h} / \beta$ et $\Pi_{1}^{h}$ est la projection $L^{2}$ de $Y$ sur les fonctions de $X_{h}$ à divergence nulle. On obtient donc deux systèmes préconditionnés :

$$
P_{h}=M_{h}^{-1} B_{h} M_{h}^{-1} A_{h} \quad \tilde{P}_{h}=M_{h}^{-1} \tilde{B}_{h} M_{h}^{-1} A_{h},
$$

dont l'analyse repose sur la proposition suivante :

Proposition 4.2. il existe $C>0$ tel que, pour $h$ suffisamment petit, les normes d'opérateurs $\left|\mathcal{A}_{h}\right|_{X X^{\star}},\left|\mathcal{B}_{h}\right|_{Y_{Y^{\star}}}$, $\left|\tilde{\mathcal{B}}_{h}\right|_{Y Y^{\star}},\left|\mathcal{M}_{h}\right|_{X Y^{\star}}$ et $\left|\mathcal{M}_{h}\right|_{Y X^{\star}}$ sont majorées par $C$, et les constantes $L B B\left[\mathcal{A}_{h}\right]_{X X^{\star}}$, $\left[\mathcal{B}_{h}\right]_{Y Y^{\star}},\left[\mathcal{M}_{h}\right]_{X Y^{\star}}$ et $\left[\mathcal{M}_{h}\right]_{Y X^{\star}}$ sont minorées par $1 / C$. Pour $\tilde{\mathcal{B}}_{h}$ on a simplement $\left[\tilde{\mathcal{B}}_{h}\right]_{Y Y^{\star}}>0$.

Preuve. Les majorations en normes d'opérateurs de $\mathcal{A}_{h}$ et $\mathcal{B}_{h}$ résultent de la continuité de $A$ et $B$, celle concernant $\mathcal{M}_{h}$, de la continuité du produit de dualité entre $X$ et $Y$, et celle de $\tilde{B}_{h}$, de la continuité de $G_{0}$ et de la majoration $\left\langle\Pi_{1}^{h} u, v\right\rangle \leq\left|\Pi_{1}^{h} u\right|\left|\Pi_{1}^{h} v\right| \leq|u|_{Y}|v|_{Y}$, valable pour tout $u, v \in X_{h}$ et uniforme en $h$. Les conditions Inf-Sup sur $\mathcal{M}_{h}$ sont équivalentes à l'hypothèse (H2) d'après la proposition 4.1.

L'opérateur $\nabla\left(G-G_{0}\right) \nabla$. est d'ordre 0 , donc continu de $X$ dans $\mathcal{S}^{1 / 2}$ et compact de $X$ dans $Y$. Quant à $G$ et $J_{2}$, ce sont des opérateurs d'ordre -1 qui sont eux aussi compacts de $X$ dans $Y$. Ainsi $A$ est une perturbation compacte de l'opérateur $A_{0}=i \alpha \nabla G_{0} \nabla \cdot / k+\beta / 2$. À des constantes multiplicatives près, on a les inégalités suivantes :

$$
\left|\left\langle A_{0} u, \bar{u}\right\rangle\right| \geq\left(G_{0} \nabla \cdot u, \nabla \cdot u\right)+|u|^{2} \geq\|u\|_{-1 / 2}^{2}+\left|\Pi_{1} u\right|^{2} \geq\left|\Pi_{2} u\right|_{1 / 2}^{2}+\left|\Pi_{1} u\right|^{2}=|u|_{X}^{2}
$$


où l'on a fait jouer pour la seconde inégalité la relation (9) et pour la dernière inégalité, la continuité $H_{\text {div }}^{-1 / 2} \rightarrow$ $H_{\mathrm{T}}^{1 / 2}$ de $\Pi_{2}$ (revenir à l'expression (4) de $\Pi_{2}$ pour s'en assurer). De même, toujours à des constantes multiplicatives près :

$$
|\langle B u, \bar{u}\rangle| \geq\left(G_{0} u, u\right)+\left(\Pi_{1} u, u\right) \geq|u|_{-1 / 2}^{2}+\left|\Pi_{1} u\right|^{2} \geq\left|\Pi_{2} u\right|_{-1 / 2}^{2}+\left|\Pi_{1} u\right|^{2}=|u|_{Y}^{2}
$$

où cette fois la continuité $H_{\mathrm{T}}^{-1 / 2} \rightarrow H_{\mathrm{T}}^{-1 / 2}$ de $\Pi_{2}$ suffit pour justifier la dernière inégalité. Puisque $X_{h}$ est stable par conjugaison, $A_{0}$ et $B$ vérifient les conditions Inf-Sup discrètes uniformes et donc aussi $A$ qui est une perturbation compacte de $A_{0}$ (d'après [6] prop. A.5.2). Enfin, on vérifie sans effort que $\left[\tilde{\mathcal{B}}_{h}\right]_{Y Y^{\star}}$ est bien strictement positif.

Remarques sur la convergence numérique de la solution de la CFIE. Si $u \in X$ est la solution exacte d'une équation CFIE s'écrivant $A u=l$ avec $l \in X^{\star}$, alors une approximation numérique $u_{h} \in X_{h}$ de cette solution vérifie $\mathcal{A}_{h} u_{h}=l_{\mid X_{h}}$. Or d'après la proposition $4.2, A$ satisfait des conditions Inf-Sup discrètes uniformes en $h$ (i.e. $\left[\mathcal{A}_{h}\right]_{X X^{\star}}$ est minorée indépendemment de $h$ ), et donc puisque $X_{h}$ approche $X$ on en déduit que $u_{h}$ converge vers $u$ dans $X$ (d'après le résultat classique de Babuška [2]). De plus, on vérifie aisément que $X=L^{2} \cap H_{\text {div }}^{-1 / 2}$, et que sa topologie coïncide avec la topologie initiale induite par les applications Id $: X \rightarrow L^{2}$ et $\operatorname{div}: X \rightarrow H^{-1 / 2}$. Autrement dit, la solution numérique de la CFIE réalise une convergence en énergie pour les courants, et en norme $H^{-1 / 2}$ pour les charges. On remarque en outre que ce résultat de convergence peut s'observer sur une classe d'éléments finis très étendue (en particulier sur les éléments de Raviart-Thomas), puisque la condition Inf-Sup qui en est le fondement ne fait appel qu'à la seule hypothèse (H0) et au fait que $X_{h}$ approche $X$. Un tel résultat de convergence est, à la connaissance de l'auteur, unique pour la CFIE, contrairement à l'EFIE qui à ce titre a été bien plus étudiée ( $c f$. par exemple [3,6] ou [8]).

\subsection{Analyse numérique des préconditionneurs}

Pour analyser le système matriciel préconditionné $P_{h}$ décrit par (14), on considère l'endomorphisme $\mathcal{P}_{h}=$ $j_{h} P_{h} j_{h}^{-1}$ de $X_{h}$, où $j_{h}$ est le morphisme intervenant dans l'expression (12). Cette même expression justifie aussi le calcul $\mathcal{P}_{h}=j_{h}\left(j_{h}^{\star} \mathcal{M}_{h} j_{h}\right)^{-1}\left(j_{h}^{\star} \mathcal{B}_{h} j_{h}\right)\left(j_{h}^{\star} \mathcal{M}_{h} j_{h}\right)^{-1}\left(j_{h}^{\star} \mathcal{A}_{h} j_{h}\right) j_{h}^{-1}=\mathcal{M}_{h}^{-1} \mathcal{B}_{h} \mathcal{M}_{h}^{-1} \mathcal{A}_{h}$ et donc pour tout $u_{h} \in X_{h}$ tel que $\left|u_{h}\right|_{X}=1$, on a les estimations suivantes :

$$
\left[\mathcal{M}_{h}^{-1}\right]_{Y^{\star} X}\left[\mathcal{B}_{h}\right]_{Y Y^{\star}}\left[\mathcal{M}_{h}^{-1}\right]_{X^{\star} Y}\left[\mathcal{A}_{h}\right]_{X X^{\star}} \leq\left|\mathcal{P}_{h} u_{h}\right|_{X} \leq\left|\mathcal{M}_{h}^{-1}\right|_{Y^{\star} X}\left|\mathcal{B}_{h}\right|_{Y Y^{\star}}\left|\mathcal{M}_{h}^{-1}\right|_{X^{\star} Y}\left|\mathcal{A}_{h}\right|_{X X^{\star}}
$$

Ainsi, grâce à la proposition 4.2, pour $h$ suffisamment petit, $\frac{1}{C^{4}}<\left|\mathcal{P}_{h} u_{h}\right|_{X}<C^{4}$. De la même façon, on montre que si $\tilde{\mathcal{P}}_{h}=j_{h} \tilde{P}_{h} j_{h}^{-1}$, alors $0<\left|\tilde{\mathcal{P}}_{h} u_{h}\right|_{X}<C^{4}$. On en déduit donc que :

Proposition 4.3. Pour $h$ suffisamment petit, les systèmes linéaires préconditionnés $P_{h}$ et $\tilde{P}_{h}$ ne sont jamais singuliers. En outre, $P_{h}$ est spectralement borné en $h$, et le rayon spectral de $\tilde{P}_{h}$ est borné en $h$.

En effet, si l'on munit $\mathbb{C}^{N(h)}$ de la norme || faisant de la bijection $j_{h}$ une isométrie, on a $\left|P_{h} \lambda\right|=\left|\mathcal{P}_{h} u_{h}\right|_{X}$ $\left(u_{h}=j_{h} \lambda\right)$ et les encadrements vrais pour $\left|\mathcal{P}_{h} u_{h}\right|$ le sont aussi pour $\left|P_{h} \lambda\right|$, permettant ainsi de conclure. Idem pour $\tilde{P}_{h}$.

Les opérateurs $\mathcal{P}_{h}$ et $\tilde{\mathcal{P}}_{h}$ éclairent aussi le lien existant entre la convergence d'une méthode itérative de type Gradient Conjugué (GC) appliquée à l'équation continue, et la convergence de ce même GC appliqué au système discret. En effet, à chaque itération le processus de Lanczos du GC dans le cas continu (resp. discret) est uniquement déterminé par la valeur du produit de l'opérateur sous-jacent à l'équation (resp. la matrice) et du résidu calculé à l'itération précédente. À ce titre, le résultat qui suit est crucial et l'on renvoie le lecteur au paragraphe 2.4 [6] pour trouver une analyse des conséquences favorables qu'entraîne une telle proposition sur le comportement des itérations.

Proposition 4.4. Si une suite $u_{h}$ d'éléments de $X_{h}$ converge vers $u$ dans $X$, alors $\mathcal{P}_{h} u_{h}$ et $\tilde{\mathcal{P}}_{h} u_{h}$ convergent vers $B A$ dans $X$. 
Lemme 4.5. Si une suite $u_{h}$ (d'éléments non nécessairement inclus dans $X_{h}$ ) converge vers $u$ dans $X$ (resp. $Y$ ), alors $Q^{h} u_{h}$ et $\Pi_{1}^{h} u_{h}$ convergent respectivement vers $u$ et $\Pi_{1} u$ dans $X$ (resp. $Y$ ).

Preuve du lemme. La projection $\Pi_{1}^{h}$ est stable sur $X$ puisqu'il existe $C>0$ tel que $C|u|_{X} \geq|u| \geq\left|Q^{h} u\right| \geq$ $\left|\Pi_{1}^{h} u\right|=\left|\Pi_{1}^{h} u\right|_{X}$. Puisque $\Pi_{1}^{h}$ est une projection au sens $L^{2}$, on en déduit, par application de la proposition 4.1, que $\Pi_{1}^{h}$ est aussi stable sur $Y$. Il suffit donc d'établir le lemme pour une suite constante $u_{h}=u$. En effet, grâce à la stabilité de $Q_{h}$ et $\Pi_{1}^{h}$, on a par exemple pour $X:\left|u-Q^{h} u_{h}\right|_{X} \leq\left|u-Q^{h} u\right|_{X}+C\left|u-u_{h}\right|_{X}$ et $\left|\Pi_{1} u-\Pi_{1}^{h} u_{h}\right|_{X} \leq\left|\Pi_{1} u-\Pi_{1}^{h} u\right|_{X}+C\left|u-u_{h}\right|_{X}$. Et idem pour $Y$.

Si $u \in X$, alors par (H1) on dispose de deux suites $\left(u_{h}\right)$ et $\left(v_{h}\right)$ de $X_{h}$ (avec $\left.\nabla \cdot v_{h}=0\right)$ qui convergent respectivement vers $u$ et $\Pi_{1} u$ dans $X$. Toujours grâce à la stabilité de $Q^{h}:\left|u-Q^{h} u\right|_{X} \leq(1+C)\left|u-u_{h}\right|_{X}$, et d'autre part : $\left|\Pi_{1} u-\Pi_{1}^{h} u\right|_{X}=\left|\Pi_{1} u-\Pi_{1}^{h}\left(\Pi_{1} u\right)\right| \leq\left|\Pi_{1} u-v_{h}\right|_{X}$. La démonstration pour $Y$ est en tout point similaire.

Preuve de la proposition 4.4. On a déjà remarqué que $\mathcal{P}_{h}=\mathcal{M}_{h}^{-1} \mathcal{B}_{h} \mathcal{M}_{h}^{-1} \mathcal{A}_{h}$ et donc en utilisant (13) et (11) il vient que $\mathcal{P}_{h}=Q^{h} \mathcal{M}^{-1}\left(i_{h}^{\star}\right)^{-1} i_{h}^{\star} B i_{h} Q^{h} \mathcal{M}^{-1}\left(i_{h}^{\star}\right)^{-1} i_{h}^{\star} A i_{h}$ et ainsi que $\mathcal{P}_{h} u_{h}=Q^{h} B Q^{h} A u_{h}$. Posons $P^{h}=1-Q^{h}$. Alors $B A u_{h}-\mathcal{P}_{h} u_{h}=B A u_{h}-Q^{h} B Q^{h} A u_{h}=P^{h} B Q^{h} A u_{h}+B P^{h} A u_{h}$ converge vers 0 dans $X$ par application du lemme 4.5. On en déduit que $\mathcal{P}_{h} u_{h}$ converge vers $B A u$. Le lemme prouve aussi que $\mathcal{P}_{h} u_{h}-\tilde{\mathcal{P}}_{h} u_{h}=\frac{2}{\beta} Q^{h}\left(\Pi_{1}-\right.$ $\left.\Pi_{1}^{h}\right) Q^{h} A u_{h}$ converge vers 0 , et donc que $\tilde{\mathcal{P}}_{h} u_{h}$ converge vers $B A u$.

\section{Conclusion}

Une paramétrix de la CFIE a été exhibée. Cette paramétrix a ensuite inspiré deux préconditionneurs pour cette équation. Dans les deux cas, il s'agit à chaque itération de réaliser le produit du résidu avec la somme d'une convolution (de noyau $1 / r$ ) et d'une version plus ou moins approchée (dépendant du préconditionneur), du premier projecteur de la décomposition de Helmholtz (projection $L^{2}$ sur les courants à divergence nulle). Le coût numérique de la synthèse de ces préconditionneurs est tout à fait compétitif, puisque la partie convolutive peut se comprimer à l'aide d'un algorithme multipôle et que la projection est réalisable en $\mathcal{O}(N)$ opérations ( $N$ étant la dimension de l'espace d'approximation).

L'analyse numérique de ces préconditionneurs nous a conduit à considérer des espaces fonctionnels originaux construits à l'aide de la décomposition de Helmholtz. La pertinence de ce choix fonctionnel est attestée par le fait qu'on peut démontrer un résultat de convergence de la solution numérique la CFIE vers la solution exacte. Mais surtout, le cadre fonctionnel retenu permet de montrer que les préconditionneurs étudiés, sous certaines hypothèses clairement identifiées concernant les espaces d'approximations, ont une action régularisante qui devrait se traduire en pratique par une accélération de la convergence des méthodes itératives de type gradient conjugué.

\section{RÉFÉRENCES}

[1] R.J. Adams, Physical and analytical properties of a stabilized electric field integral equation. IEEE Trans. Antennas Propag. 52 (2004) 362-372.

[2] I. Babuška, Error bounds for the finite element method. Numer. Math. 16 (1971) 322-333.

[3] A. Bendali, Numerical analysis of the exterior boundary value problem for the time harmonic maxwell equations by a boundary finite element method. Part 2: The discrete problem. Math. Comp. 43 (1984) 47-68.

[4] A. de La Boudonnaye, Décomposition $H_{\text {div }}^{-1 / 2}(\Gamma)$ et nature de l'opérateur de Steklov-Poincaré du problème extérieur de l'électromagnétisme. C. R. Acad. Sci. Paris, Sér. I 316 (1993) 369-372.

[5] Q. Carayol, Développement et analyse d'une méthode multipôle multiniveau pour l'électromagnétisme. Ph.D. Thesis, Université Paris VI (2002).

[6] S. Christiansen, Résolution des équations intégrales pour la diffraction d'ondes acoustiques et électromagnétiques. Stabilisation d'algorithmes itératifs et aspects de l'analyse numérique. Ph.D. Thesis, École Polytechnique (2001).

[7] R. Colton and D. Kress, Integral Equation Methods in Scattering Theory. John Wiley \& Sons (1983).

[8] I. Ivakhnenko, Yu.G. Smirnov and E.E. Tyrtyshnikov, The electric field integral equation: theory and algorithms. Inst. Math. Appl. Conf. Ser. New Der. 65 (1998) 251-262. 
[9] D.P. Levadoux, Étude d'une équation intégrale adaptée à la résolution hautes fréquences de l'équation de Helmholtz. Ph.D. Thesis, Université Paris VI (2001).

[10] D.P. Levadoux and B.L. Michielsen, A new class of integral equations for scattering problems. Sixth International Conference on Mathematical and Numerical Aspects of Wave Propagation, Jyvaskyla, June 30-July 4 (2003).

[11] D.P. Levadoux and B.L. Michielsen, Nouvelles formulations intégrales pour les problèmes de diffraction d'ondes. ESAIM: $M 2 A N 38$ (2004) $157-175$.

[12] W. McLean and T. Tran, A preconditioning strategy for boundary element galerkin methods. Numer. Methods Partial Differential Equations 13 (1997) 283-301.

[13] B. Michielsen and D.P. Levadoux, Application de méthodes topologiques pour la réalisation de modèles CEM prédictifs dans le domaine hyperfréquence. Technical Report RF 2/03038 DEMR/DR, ONERA (Décembre 2002).

[14] J.-C. Nédélec and J. Planchard, Une méthode variationnelle d'éléments finis pour la résolution numérique d'un problème extérieur dans $\mathbb{R}^{3}$. RAIRO Sér. Rouge 7 (1973) 105-129.

[15] P.A. Raviart and J.-M. Thomas, A mixed finite element method for 2nd order elliptic problems, in Mathematical aspects of the finite element method, I. Galligani, E. Magenes Eds., Springer-Verlag, Lect. Notes Math. 606 (1977) 292-315.

[16] J. Simon, Extension de méthodes multipôles rapides : résolution pour des seconds membres multiples et application aux objets diélectriques. Ph.D. Thesis, Université de Versaille Saint-Quentin-en-Yvelines (2003).

[17] O. Steinbach, On the stability of the $L_{2}$ projection in fractional Sobolev spaces. Numer. Math. 88 (2001) 367-379.

[18] O. Steinbach, On a generalized $L_{2}$ projection and some related stability estimates in Sobolev spaces. Numer. Math. 90 (2002) $775-786$.

[19] O. Steinbach and W.L. Wendland, The construction of some efficient preconditioners in the boundary element method. Adv. Comput. Math. 9 (1998) 191-216.

[20] G. Sylvand, La méthode multipôle rapide en électromagnétisme : performance, parallélisation, applications. Ph.D. Thesis, École Polytechnique (2002).

[21] D. Volpert and D.P. Levadoux, Expertise SER et code axisymétrique pour objets de révolution. Technical Report 1/05592 DEMR, ONERA (Décembre 2001).

To access this journal online:

www.edpsciences.org 